\title{
Effect of relay nodes and transmit power on end-to-end delay in multi-hop wireless ad hoc networks
}

\author{
Research Lab, \\ Electrical Engineering Department, \\ IIT Hyderabad, Yeddumailaram, \\ ODF Estate, Medak District-502205, \\ Andhra Pradesh state, India \\ E-mail: ee10p004@iith.ac.in \\ E-mail: raji@iith.ac.in \\ *Corresponding author
}

Raja Vara Prasad Yerra* and P. Rajalakshmi

\begin{abstract}
Channel access delay in a wireless multi-hop ad hoc network is the major source of delay while considering end-to-end delay. In this work, end-to-end delay is analysed considering silent relay nodes and effect of network parameters like node density and throughput. Given network parameter requirements and bound on end-to-end delay, optimal ranges of transmission radius and channel access probability can be obtained from the proposed analysis. Effect of silent relay nodes must be considered to maintain quality of service $(\mathrm{QoS})$ metrics. Transmission power adaptability to reduce end-to-end delay is analysed considering the interference model. Increase in transmission power is not reducing end-to-end delay linearly. Simulation results show that increase in end-to-end delay due to channel access probability and throughput is considerably higher than node density. Also given the network parameters, end-to-end delay can be minimised only up to certain value irrespective of increase in transmit power.
\end{abstract}

Keywords: silent relay nodes; channel access probability; node density; end-to-end delay; interference model; ad hoc networks; multi-hop.

Reference to this paper should be made as follows: Yerra, R.V.P. and Rajalakshmi, P. (2014) 'Effect of relay nodes and transmit power on end-to-end delay in multi-hop wireless ad hoc networks', Int. J. Space-Based and Situated Computing, Vol. 4, No. 1, pp.26-38.

Biographical notes: Raja Vara Prasad Yerra received his MTech from IIT Kharagpur and pursuing his PhD in Wireless Communication Systems from IIT Hyderabad. He has been working for different sponsored projects like pervasive sensor networks, cyber physical systems and Damoder Valley Corporation project in last five years. His areas of interests are wireless sensor networks (WSN), cyber physical system-based (CPS) automation systems and wireless protocols for smart buildings and smart grids.

P. Rajalakshmi received his MTech and PhD from Indian Institute of Technology Madras, both in Communication Systems. She is currently working as an Assistant Professor in the Department of Electrical Engineering at Indian Institute of Technology Hyderabad. He has around ten years of research and development experience in the broad areas of wireless communications, sensor networks, embedded systems and optical networks. She has over 20 refereed journal publications and conference publications. She has been actively involved in various government sponsored projects from DST and DIT like pervasive sensor networks, IoT-eHealth, cognitive radio networks, cyber physical systems, IoT-smarter for healthcare at IITH.

This paper is a revised and expanded version of a paper entitled 'Effect of relay nodes on end-to-end delay in multi-hop wireless ad hoc networks' presented at Ninth International Workshop on Heterogeneous Wireless Networks (HWISE 2013), 26th IEEE International Conference on Advanced Information Networking and Applications, Workshops, AINA-2013, Barcelona, Spain, 25-28 March 2013.

\section{Introduction}

Ad hoc networks can communicate without fixed infrastructure. Ease and less time for deployment are the main advantages of ad hoc networks. The ad hoc networks are used in very diverse region of application from biological spheres to ubiquitous computing. Wireless sensor-based ad hoc networks have low bandwidth and more channel access delays. Delay is considered to be one 
of the main quality of service (QoS) metric for time constrained applications in ad hoc networks. Wireless Sensor networks is one of major applications nowadays as they operate mostly in ad hoc mode. Diverse delay requirements are required to support heterogeneous sensor network applications. Applications like detection of fire in forest, seismic activity need delay differentiation for best use of the measurements obtained by sensors. Delays in a wireless network can be due to three main sources

1 Delay due to multi-hop: Message traverses several hops before reaching destination in multi-hop networks.

Transmission power is analogous to transmission radius $(r)$ so low power operation of nodes in battery operated sensor networks also increases the number of hops in the network which is another reason for larger delays. Transmission power can be increased to reduce the hops but not at the cost of more interference with the neighbouring nodes.

2 Channel access delay: The channel access mechanisms are mostly CSMA/CA-based contention access in wireless networks. Collisions creates additional exponential delays in the network. Channel access delays depends on throughput of each node, node density and number of nodes in the network and transmission power.

3 Aggregation and queuing delays at intermediate nodes: Aggregation and compression in ad hoc networks is used to reduce the redundancy of the messages thereby reducing the channel access delays. Aggregation and compression function of throughput and protocols at intermediate nodes can lead to large delays due to processing delays before transmission.

These three sources of delay are tightly coupled and should be optimised considering all the above factors. The delay experienced by a message due to network throughput, node density, silent relay nodes and transmission power is analysed using simple models to understand trade-off among them in this paper. Increase in transmission power reduces the channel access delay while a higher transmission power propagates the information to a larger subset of nodes leading to more interference. There exists a tradeoff between power control and MAC layer control to provide small delays in the transmission of messages. As a result, understanding the relationship between delay and the network parameters is an important first step in providing delay differentiation. Aggregation and queuing delays are ignored in this paper.

\section{Related work}

Optimal network throughput is studied considering the effect of transmission power in Gupta and Kumar (2000) and is shown that it is obtained at the lowest transmission power that allows connectivity. The interference caused is very little with smaller transmission ranges. Lower transmission power increases number of hops $(n)$ to the destination which intern increases the delay and scales down the throughput to $1 / \sqrt{(n)}$ according to Grossglauser and Tse (2001). Trade-off exists between delay experienced and achievable throughput in the network as shown in El Gamal et al. (2004) and Narasimhan and Kunniyur (2005), but the effect of collisions and channel access mechanism are ignored in both the analysis.

Energy minimisation using routing strategy is discussed in Kar et al. (2003). Different network parameters effect on energy is not considered in this work.

MAC layer protocols (Rozovsky and Kumar, 2001; Singh and Raghavendra, 1998; Ye et al., 2002) and power control protocols (Gomez et al., 2003; Heinzelman et al., 2000; Kawadia and Kumar, 2003; Narasimhan and Kunniyur, 2005) have been proposed in the literature to provide better throughput and energy efficiency. The CSMA/CA protocol is the most common MAC layer protocol in the 802.11 standard of the IETF. The basic access mechanism, called the distributed coordination function (DCF), is a carrier sense multiple access with collision avoidance mechanism (CSMA/CA). DCF initialises a random countdown timer and senses the medium. Contention in the medium causes more end-to-end delay due to exponential back-off.

Increase in transmission power decreases number of hops thereby reducing the total end-to-end delay of a multi-hop route. However increase in transmission power also increases the interference among neighbour nodes. A dynamic power adjustment scheme is proposed in Rukpakavong et al. (2011) with neighbour discovery using RSSI value. Analysis in Rukpakavong et al. (2011) considers only single hop network considering only packet loss and energy consumption. Transmit power adjustment with environmental changes is discussed in Pianegiani (2011). Interference, link quality and energy consumption is considered in Pianegiani (2011), but other network parameters like node density, throughput and channel access probability are ignored. A new power management scheme based on path loss model for 802.15.4 is implemented in $\mathrm{Fu}$ and Ma (2009). Algorithm in Fu and Ma (2009) claims extended battery life without considering the effect of network parameters. Transmission power control (TPC) with binary search algorithm is discussed in Oh (2012). meeIEEE 802.15.4 MAC is modified implementing TPC adjusting to environmental conditions using path loss model. Work presented in Oh (2012) does not consider the effect of network parameters. In Lin et al. (2006), optimal transmission power is estimated using RSSI and LQI, and reflected changes in link according to the time by adopting the feedback scheme. Limitation of this approach is that if the number of nodes participating in a network increases, measuring of RSSI would become difficult due to interference. Son et al. (2004) and Kawadia et al. (2001) attempted to reduce energy consumption with a single transmission power being used by all nodes in a network and proposed a solution scheme at the network-level. There are recent advancements in mobile ad hoc networks routing protocols as mentioned in Palmieria and Castiglione (2012). 
A dynamic adaptive routing protocol based on condensation phenomenon is proposed ensuring throughput and end-to-end delay. Effect Of relay nodes. On end-to-end delay in multi-hop wireless ad hoc networks is well analysed considering network parameters in our previous work (Prasad and Rajalakshmi, 2013). Throughput and end-to-end delay parameters of consideration in Palmieria and Castiglione (2012) are similar to our work in Prasad and Rajalakshmi (2013). Almost all of these studies have focused on obtaining better trade-off between accuracy and cost, and aimed to make precise control on reduced energy consumption in transmitting packets.

Relay nodes and transmission power plays an important role in reducing end-to-end delay of wireless multi-hop network. Increase in interference causes more collisions and more contention for the channel. In order to mitigate the interference appropriate interference model needs to be considered for reliable reception of a packet. According to this model neighbourhood nodes should remain silent for the present transmission of the node. Increase in transmission power increase the number of neighbourhood nodes, which also causes the relay nodes in the simultaneous multi-hop route to be silent for a longer duration. Hence even though there is increase in transmission power for a set of nodes in one multi-hop route to reduce the end-to-end delay, it may increase the end-to-end delay of other simultaneous routes as there may be silent relay nodes in neighbourhood nodes set. Tradeoff between the transmission power and interference need to be analysed for a better understanding of the network. In this paper a silent relay node concept is introduced first and explained in detail. Effect of transmission power increase on the end-to-end delay is also analysed. End-to-end delay variation with network parameters like node density, throughput and channel access probability is simulated in detail and explained in further sections of this paper.

The rest of the paper is organised as follows. Section 2 describes the system model with silent relay nodes. In Section 3 effect of silent relay nodes on end-to-end delay is discussed and Section 4 presents the simulation results on end-to-end delay with silent relay nodes. Section 5 describes the effect of increase in transmit power versus end-to-end delay and Section 6 concludes the paper.

\section{System model with silent relay nodes}

The end to end delay in multi-hop ad hoc network is mainly due to channel access delay and queuing delays. In this paper effect of silent relay node delay is considered on the end-to-end delay of multi-hop network. Effect of network parameters on the end-to-end delay is analysed in detail considering tradeoff between parameters. The parameters considered for analysis are transmission radius $(r)$, channel access probability $(p)$, node density $(\Lambda)$ and percentage of silent relay nodes $(\alpha)$. Expected channel access delay is derived in Narasimhan and Kunniyur (2005) given as in equation (1). Where $f$ is the neighbourhood radius as given in equation (2). The total end to end delay for a message to travel L units away is also derived in Narasimhan and Kunniyur (2005) as given in equation (4). Silent relay node is introduced in detail in the next subsection of the paper.

$$
\begin{aligned}
& E\left[d_{c}\right]=\frac{e^{-\Lambda \pi f^{2}}}{p}\left[e^{\frac{\Lambda \pi f^{2}}{1-\frac{p \lambda D}{r}}}-1\right] \\
& f=(1+\Delta) r \\
& d_{t}=\sum_{j=1}^{n} d_{c}(j) \\
& E\left[d_{t}\right]=\frac{L e^{-\Lambda \pi f^{2}}}{p * r}\left[e^{\frac{\Lambda \pi f^{2}}{1-\frac{p \lambda D}{r}}}-1\right]
\end{aligned}
$$

$\Delta \quad$ is number of neighbour hops to be silent during transmission. Nodes to be silent is take $n$ as two hops for overlapping regions.

$\Lambda$ is the node density.

\subsection{Silent relay node}

Channel access probabilities in a multi-hop network is assumed to be independent and same in magnitude for all relay nodes in the literature. The above assumption is not valid for all types of relaying nodes. Relay nodes which are common for more than one multi-hop route and silent relay nodes during others transmission have different channel access delays. Three types of relay nodes are considered for analysis as shown in Figure 1. The node R-2 shown in Figure 1 is common relay node for three multi-hop routes. R-1 and R-2 relay nodes are silent relay nodes and remaining relay nodes are normal relay nodes which just forward data. Silent relay node is the relay node that will remain silent during other nodes transmission when it is present in other nodes transmission region. In this paper total end-to-end delay is analysed with silent relay nodes and common congested relay nodes are ignored for the sake of simplicity.

Figure 1 Pictorial representation of types of relay nodes in multi-hop ad hoc network (see online version for colours)

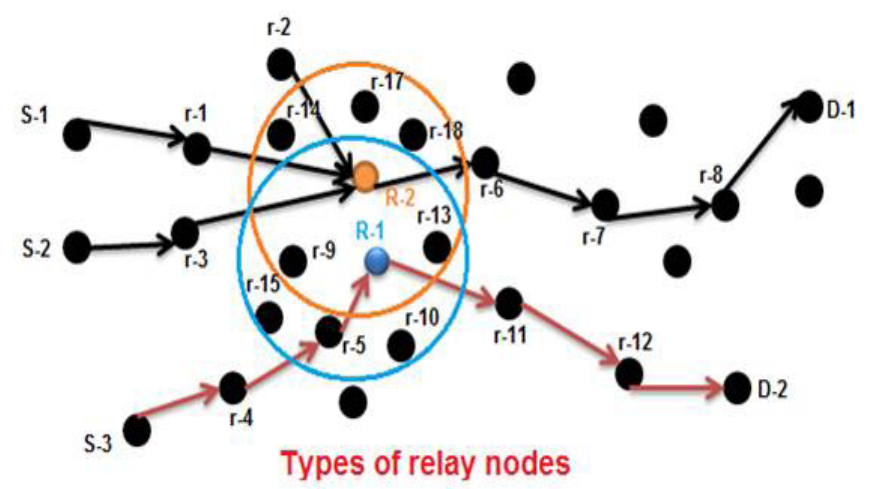

Nodes in a multi-hop network can transmit data with a certain transmission power to reach the next hop. The nodes 
in this transmission region $(r)$ can hear this data transmission. The nodes in this transmission region should remain silent when one node is transmitting. Nodes r-9, 13, $14,17,18, \mathrm{R}-1$ should remain silent for $\mathrm{R}-2$ relay node transmission and nodes $\mathrm{r}-5,9,10,13,15, \mathrm{R}-2$ should remain silent for R-1's transmission. The interference model is similar to Protocol model in Gupta and Kumar (2000) when one node is transmitting the nodes that should remain silent is given as shown in equations (5) and (6).

If the nodes $i$ and $j$ are within a distance $r$ of each other and $k$ is any other node

$$
\begin{aligned}
& d(i, j) \leq r \\
& d(i, k) \leq(1+\Delta) r
\end{aligned}
$$

for some $(\Delta>0)$

Equation (5) indicates that node $\mathrm{j}$ to be within the transmission range of node $i$. The equation (6) requires a $(1+\Delta) r$ neighbourhood of node $i$ to be silent for successful transmission. $\Delta=0$ is the condition for only one hop neighbour nodes to be silent but as shown in Figure 1 the relay nodes R-1 and R-2 of different multi-hop routes are in each others transmission range. The nodes that should remain silent for R-1's transmission is $\mathrm{r}-5, \mathrm{r}-9, \mathrm{r}-10, \mathrm{r}-13$, $\mathrm{r}-15$ and R-2. In similar way the nodes that should remain silent for R-2's transmission is r-9, 13, 14, 17, 18 and R-1. Nodes r-14, r-17, r-18 should remain silent for R-1's transmission even though there are not in the transmission region of $\mathrm{R}-1$. This is because the relay node $\mathrm{R}-2$ which is in the silent region cannot accept packets from these nodes. The end-to-end delay increases due to silent R-2 node even though the nodes $\mathrm{r}-14, \mathrm{r}-17, \mathrm{r}-18$ are not in the transmission region of R-1 virtually making second hop neighbourhood to be silent $(\Delta=1)$. The effect of silent relay nodes $(\alpha)$ along with network parameters like node density $(\Lambda)$, throughput $(\lambda)$, and channel access probability $(p)$ is discussed in this paper. Percentage of silent relay nodes is assumed to be $\alpha$ among the total relay nodes to find the end-to-end delay.

\section{Effect of silent relay nodes on end-to-end delay}

The channel access delay in the single hop neighbourhood is considered in Narasimhan and Kunniyur (2005). The underlying assumption in Narasimhan and Kunniyur (2005) is that channel access delay at each hop is independent and same magnitude. The channel access delay experienced by relay nodes in sparsely distributed region and the nodes in the overlapping transmission regions can be different. Relay nodes in overlapping transmission region remains silent for other relay nodes transmission. The percentage of relay nodes that are silent in a multi-hop route decides the amount of end-to-end delay. The percentage of number of relay nodes in overlapping transmission regions in multi-hop route to the destination is considered in calculating the end-to-end delay analysis. The effect of silent relay nodes is explained in more detail in next paragraph with help of pictorial representation of nodes as shown in Figure 2.

- $\quad$ route-1 is between nodes S-1 and D-1

- $\quad$ route-2 is between nodes S-2 and D-2

- $\quad$ route-3 is between nodes S-3 and D-3

- $\quad$ route-4 is between nodes S-4 and D-4.

Figure 2 Topology of nodes with relay nodes in overlapping transmission region (see online version for colours)

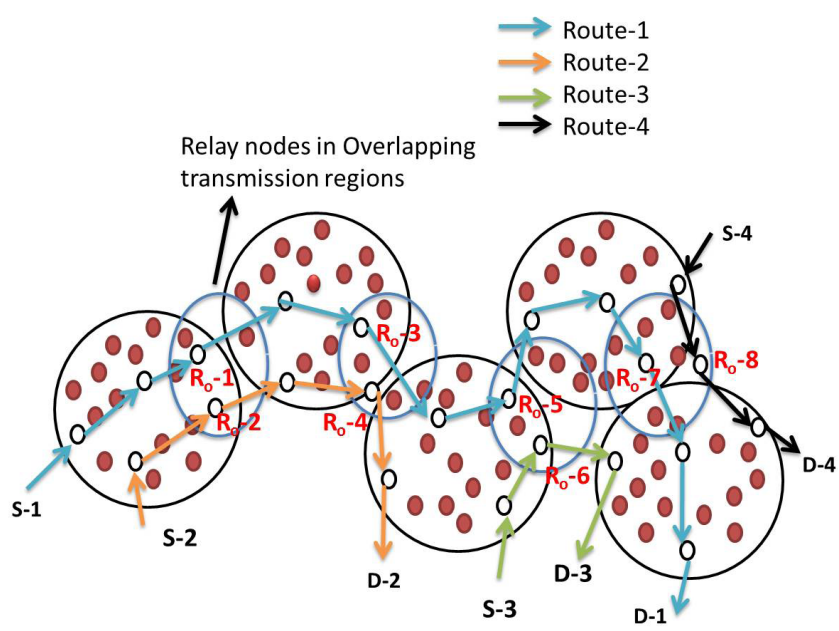

An example node distribution of ad hoc wireless network is shown in Figure 2. Four simultaneous multi-hop routes with different number of relay nodes per route is shown in Figure 2. Nodes in white colour shown in Figure 2 are relay nodes of multi-hop routes where each route indicated with different colour arrows. Two types of relay nodes are shown in Figure 2. Some of the relay nodes are present in bigger circles and some of them in the smaller circles. Relay nodes in the bigger circles are not in the vicinity of any other relay nodes where as the relay nodes shown in the smaller circle are in the one-hop transmission range of other relay node of different route. For example the Ro-1 is in the transmission range of $R_{o-2}$ of route-2. The assumption here is that preceding relay node of $R_{o-1}$ cannot hear from $R_{o-2}$ and vice-versa.

The delay for normal relay nodes is same as mentioned in literature (Narasimhan and Kunniyur, 2005) derived in equation (4). Other nodes should remain silent to minimise interference in one-hop transmission region when any node is transmitting according to Protocol model in Gupta and Kumar (2000). For example if we consider relay nodes $R_{o-1}$ and $R_{o-2}$ both are in one others transmission region, relay node $R_{o-1}$ should remain silent for $R_{o-2}$ transmission and vice versa. Average end-to-end delay increases for each route as the relay nodes are in the two hop silent region $(\Delta=1)$ to minimise the interference. The end-to-end delay considering silent relay nodes is derived in equation (13). The percentage of silent relay nodes $(\alpha)$ is formulated as shown below. 
From Figure 2 in multi-hop route-1 (S-1 to D-1)

- $\quad$ total relay nodes $(\mathrm{T})=10$

- number of relay nodes with $\Delta=1$ (two hop neighbour hood silent nodes) for route-1(t) $=4$.

Hence the percentage $(\%)$ of silent relay nodes for any route is as below

$$
\alpha=(t / T)
$$

Thus $\alpha$ for four multi-hop routes route-1, 2, 3, 4 shown in Figure 2 are $0.4,0.4,1,1$ respectively. $\alpha$ is used in calculating total end-to-end delay. End-to-end delay of relay nodes in the bigger circles are considered in equation (8) with $\Delta=0$. End-to-end delay of silent relay nodes with $\Delta=1$ is given in equation (10). Total end-to-end delay of all relay nodes is given in equation (13) by combining equations (8) and (10).

$$
\begin{aligned}
& E_{1}\left[d_{t}\right]=\frac{(1-\alpha) e^{-\Lambda \pi f_{1}^{2}}}{p * r}\left[e^{\frac{\Lambda \pi f_{1}^{2}}{1-\frac{p \lambda D}{r}}}-1\right] \\
& f_{1}=(1+\Delta) r
\end{aligned}
$$

$\Delta=0$ for relay nodes in non-overlapping transmission regions

$$
\begin{aligned}
& E_{2}\left[d_{t}\right]=\frac{\alpha e^{-\Lambda \pi f_{2}^{2}}}{p * r}\left[e^{\frac{\Lambda \pi f_{2}^{2}}{1-\frac{p \lambda D}{r}}-1}\right] \\
& f_{2}=(1+\Delta) r
\end{aligned}
$$

$\Delta=1$ for relay nodes in overlapping transmission regions.

Total end-to-end delay is obtained by combining equations (8) and (10)

$$
E\left[d_{t}\right]=E_{1}\left[d_{t}\right]+E_{2}\left[d_{t}\right]
$$

$$
\begin{aligned}
& E\left[d_{t}\right]=\frac{(1-\alpha) e^{-\Lambda \pi f_{1}^{2}}}{p * r}\left[\begin{array}{l}
\frac{\Lambda \pi f_{1}^{2}}{1-\frac{p \lambda D}{r}}-1 \\
e^{1}
\end{array}\right] \\
& +\frac{\alpha e^{-\Lambda \pi f_{2}^{2}}}{p * r}\left[e^{\frac{\Lambda \pi f_{2}^{2}}{1-\frac{p \lambda D}{r}}}-1\right]
\end{aligned}
$$

Thus end-to-end delay experienced by a message in a wireless ad hoc networks is derived taking into effect the percentage of silent relay nodes $(\alpha)$.

\section{Results on end-to-end delay with silent relay nodes}

The total end-to-end delay in a multi-hop network is primarily dependent on channel access delay at each relay node. Channel access delay derived in Narasimhan and Kunniyur (2005) is a function of channel access probability $(p)$, transmission radius $(r) /$ power and other network parameters like node density $(\Lambda)$ and throughput $(\lambda)$. The channel access delay with variation in distance $(r)$ for different channel access probability is simulated as shown in Figure 3. The optimal minimum distance for each channel access probability is different. The channel access delay $\left(E\left[d_{c}\right]\right)$ for each given channel access probabilities for equation (1) is as shown in Figure 3. This region indicates that channel access delay can be kept low with a given $p$ value up-to certain transmission radius where the delay again start increasing. This transmission radius range is different for different $p$ values as shown in Figure 3. For example if we take bound of 15 units on channel access delay the distance $(r)$ is 0.6 for $p=0.4$ and 0.7 for $p=0.15$. Increase in channel access probability $(p)$ will increases the contention among nodes. To meet the same delay requirements transmission power/ radius should be reduced to reduce the number of contending nodes.

Figure 3 Channel access delay with variation in distance $(r), \Lambda=100, \lambda=0.025, \Delta=0$ for set of channel access probabilities $(p)$ (see online version for colours)

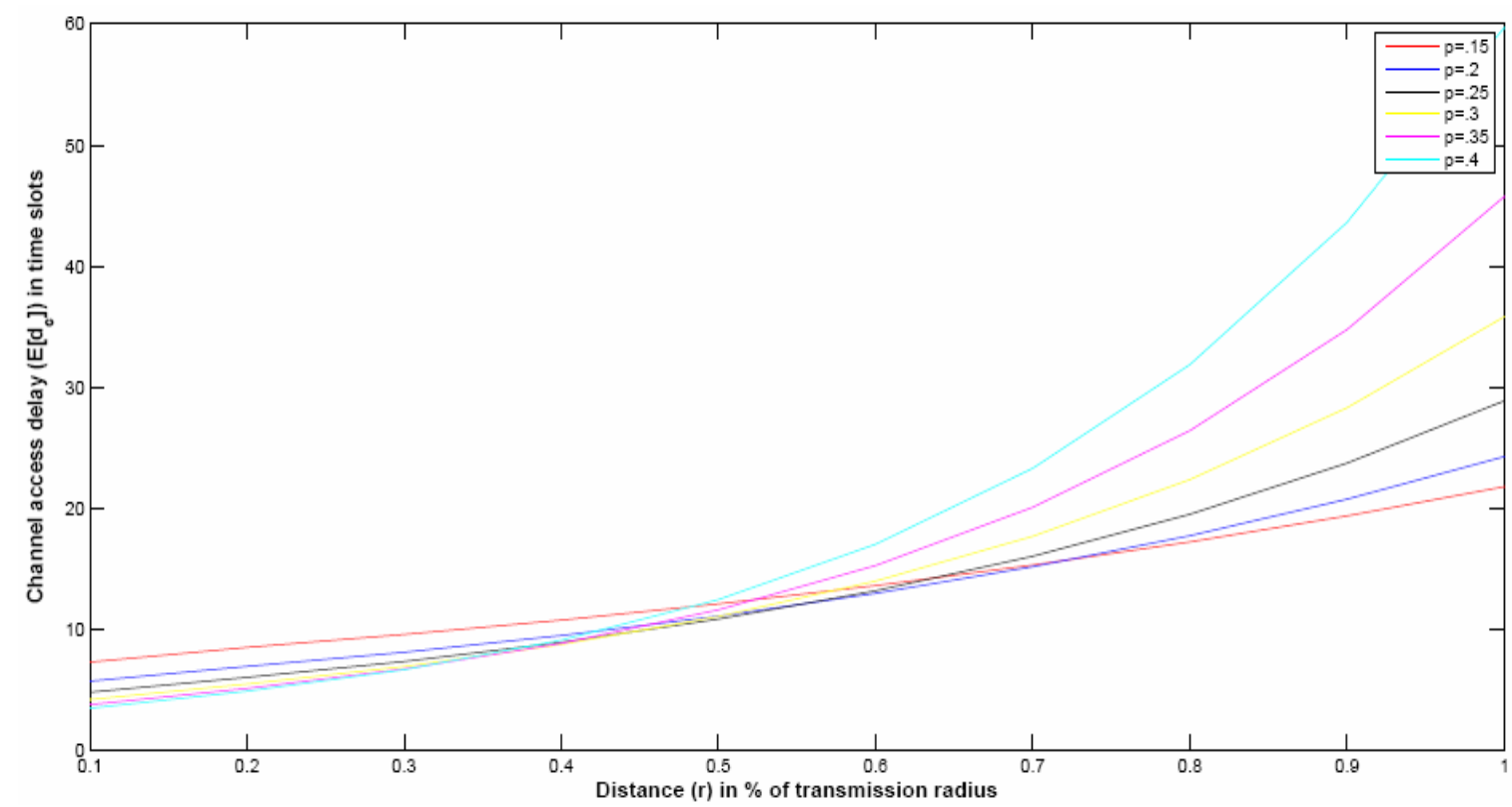


Figure 4 End-to-end delay with variation in distance $(r)$, (a) $\Lambda=100, \lambda=0.025, \Delta=0$ (b) $\Lambda=100, \lambda=0.025, \alpha=0.2$ and $\Delta=1$ for set of channel access probabilities $(p)$ (see online version for colours)

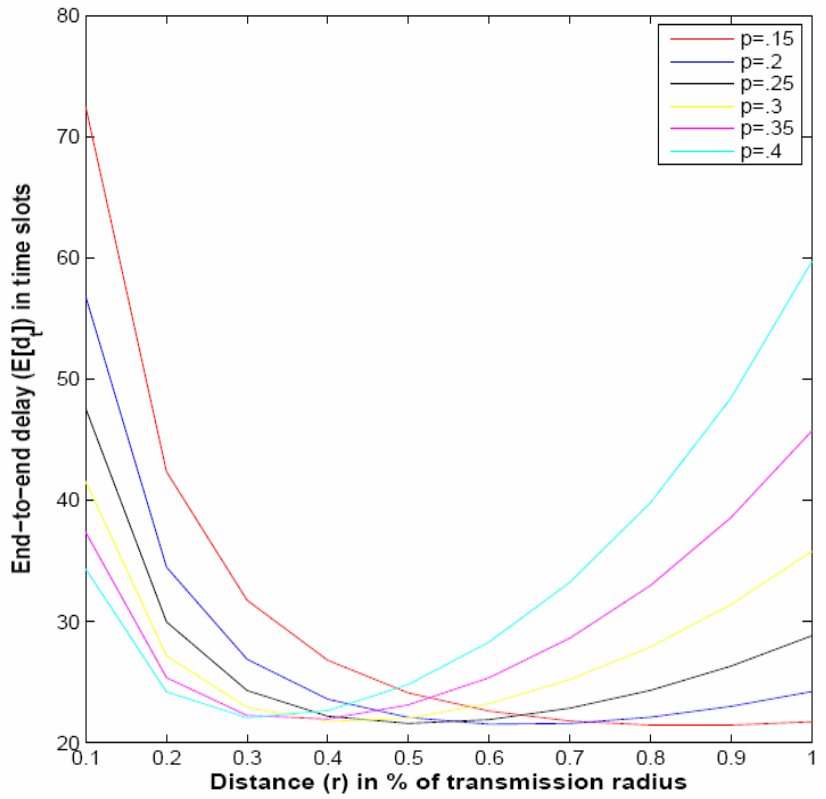

(a)

End-to-end delay in a multi-hop network is given in equation (6), where the channel access probability is assumed independent at each hop so the end-to-end delay is $\mathrm{L} / \mathrm{r}$ hop times of channel access delay. This assumption is not valid as explained in the previous sections and a new end-to-end delay equation is derived as shown in equation (13). End-to-end delay is simulated for both cases with and without silent relay nodes as shown in Figure 4. The end-to-end delay is high initially and has minimum delay region at the middle and starts increasing at the end as shown in Figure 4(a). The total end-to-end delay is effected considerably after considering the silent relay nodes compared to the previous literature results as shown in Figure 4(a). The slope of curves are steeper in Figure 4(b) with silent relay nodes. The transmission distance $(r)$ for which the end-to-end delay is minimum also reduced drastically. The distance range for channel access probability of $p=.15$ is $[00.55]$ and $[0,1]$ for with and without silent relay nodes respectively. Considering $20 \%$ of silent relay nodes among total relay nodes the end-to-end delay increased drastically as the number of nodes to remain silent increased to two hops. The transmission distance $(r)$ for a given end-to-end delay of 50 units and $p=0.15$, reduced to $45 \%$ of the previous case where there are no silent relay nodes. The distance $(r)$ for higher probabilities is still decreasing as the silent relay nodes are adding further delay to the contending nodes in the region.

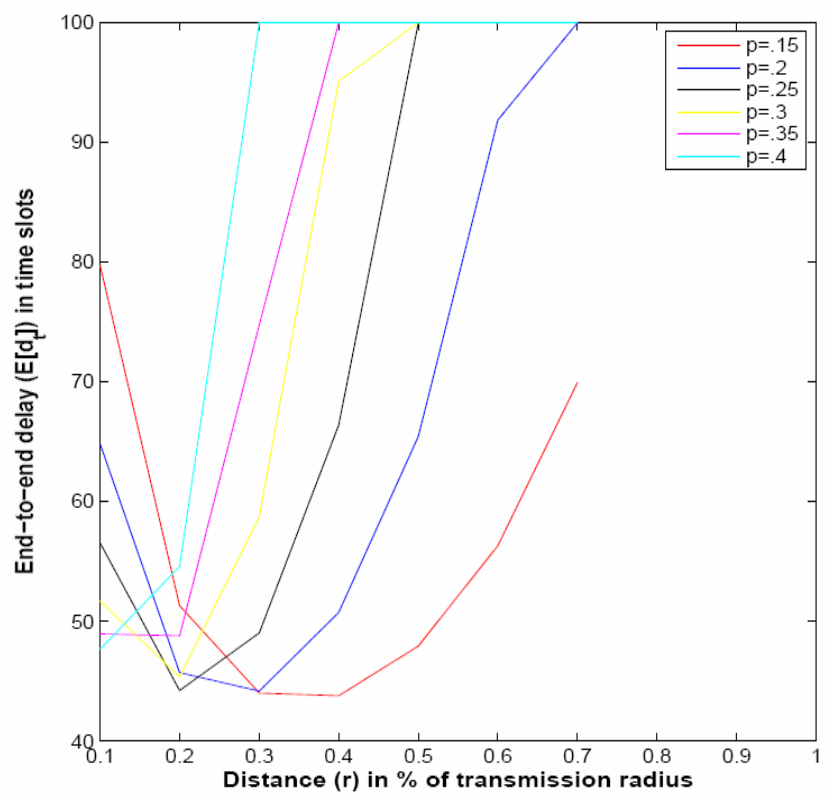

(b)

End-to-end delay is a function of other network parameters as shown in equation (13). Total end-to-end delay is analysed with respect to changes in network parameters distance $(r)$, channel access probability $(p)$ and percentage of silent relay nodes $(\alpha)$ while $\Lambda$ and $\lambda$ are fixed to 100 and 0.025 as shown in Figure 5. End-to-end delay variation with respect to simultaneous network parameters $p$ and $r$ is shown as 3D plot. The 3D surface plot looks like a valley where there is a minimal end-to-end delay region. Given a bound on end-to-end delay and fixing the network parameters optimal transmission distance $(r)$ and channel access probability $(p)$ ranges can be obtained. For example if the $\left[E\left[d_{t}\right], \Lambda, \lambda, \alpha\right]$ are fixed to $[30,100,0.025,0.2]$, the distance $(r)$ and channel access probabilities $(\mathrm{p})$ ranges are $\left[\begin{array}{ll}0.1 & 0.7\end{array}\right],\left[\begin{array}{ll}0 & 0.9\end{array}\right]$ respectively with silent relay nodes shown in Figure 5(b) and [0 0.9 ], [ [ 0.9 ] without silent relay nodes as shown in Figure 5(b). The transmission distance $(r)$ range reduced considerably due to $20 \%$ overlap region of relay nodes compared to absence of silent relay nodes. The neighbourhood hop nodes remains silent which increases the end-to-end delay and decreases the valley region as shown in Figure 5(b). Thus we have analysed the end-to-end delay with variation in distance $(r)$ and channel access probability $(p)$ in this section. In the next subsection analysis with respect to varying node density $(\Lambda)$ is considered. 
Figure $53 \mathrm{D}$ plot of end to end delay with variation in distance ( $r$ ) and channel access probability ( $p$ ), (a) $\Lambda=100, \lambda=0.025, \Delta=0$ without silent relay nodes (b) $\Lambda=100, \lambda=0.025, \Delta=1, \alpha=0.2$ with silent relay nodes (see online version for colours)

5 (a) $E\left[d_{t}\right]$ (without silent relay nodes) vs $p \& r, \Lambda=100, \lambda=0.025, \Delta=0, \alpha=0$

$5(b) E[d]$ (with silent relay nodes) vs $p \& r, \Lambda=100, \lambda=0.025, \Delta=1, \alpha=0.2$

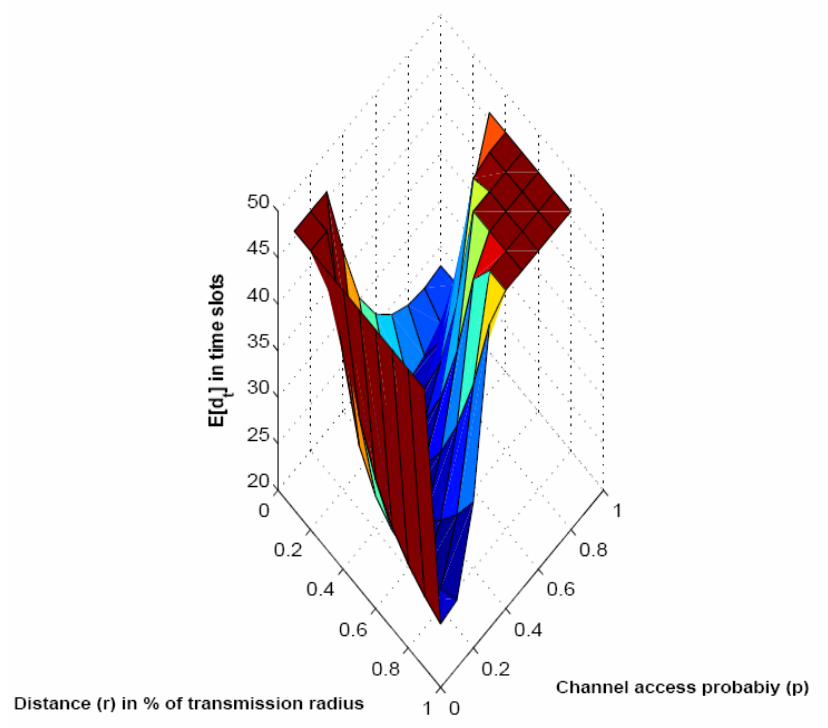

(a)

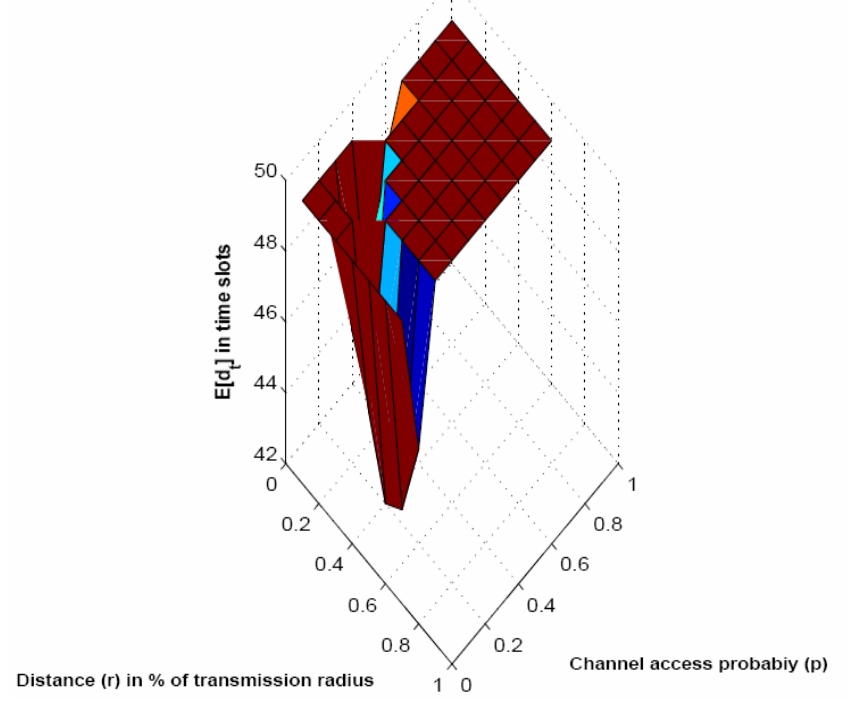

(b)

Figure $63 \mathrm{D}$ plot of end to end delay with variation in distance ( $r$ ) and channel access probability ( $p$ ) along with, (a) $\Lambda=50, \lambda=0.025$, $\Delta=0$ without silent relay nodes b) $\Lambda=50, \lambda=0.025, \Delta=1, \alpha=0.2$ with silent relay nodes (see online version for colours)

6(a) $E\left[d_{t}\right]$ (without silent relay nodes) vs $p \& r, \Lambda=50, \lambda=0.025, \Delta=0, \alpha=0$

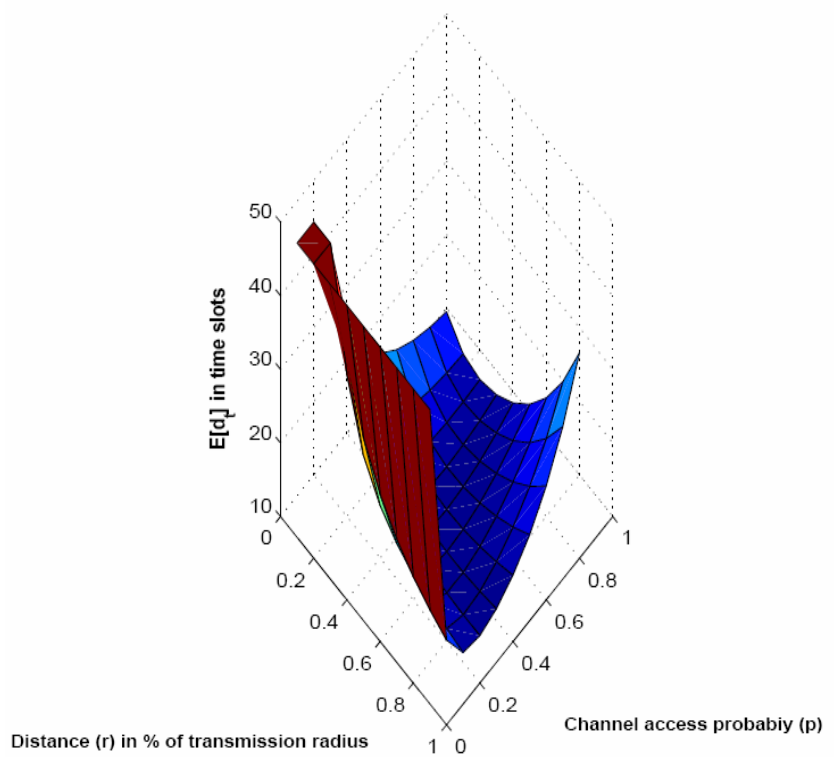

(a)

\subsection{Effect of node density $(\Lambda)$ on end-to-end delay with silent relay nodes}

Simulation results explained in previous paragraph is the analysis of end-to-end delay with variation in distance $(r)$ and channel access probabilities $(p)$ by fixing some of the parameters like node density $(\Lambda)$, throughput $(\lambda)$ to 100 and 0.025 . The effect of varying node density $(\Lambda)$ in steps of 50 , 150 and 200 with $\lambda=0.025, \alpha=0.2$ is discussed in this section. Node density is an important parameter which 6(b) $E\left[d_{t}\right]$ (with silent relay nodes) vs p \& $r, \Lambda=50, \lambda=0.025, \Delta=1, \alpha=0.2$

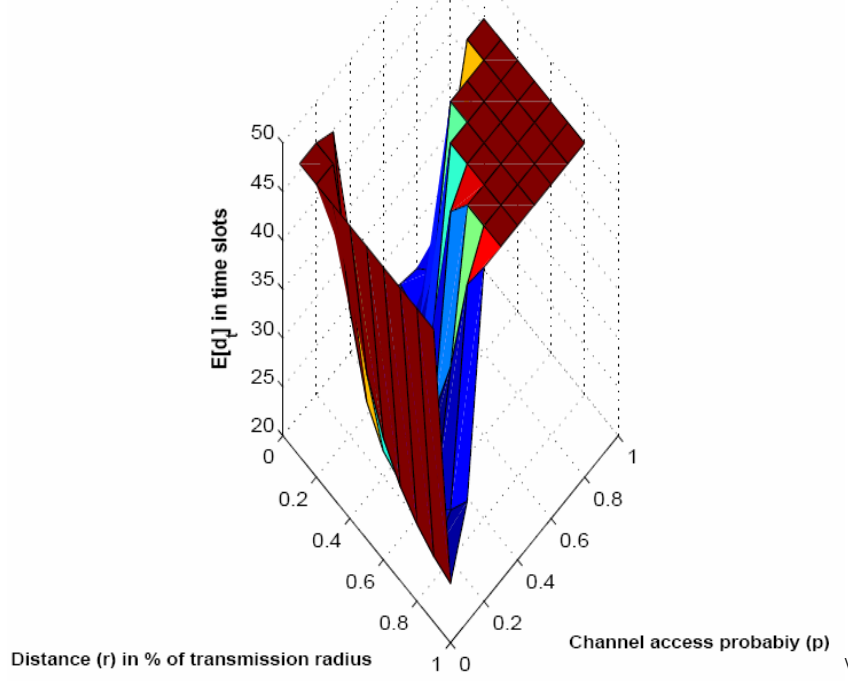

(b)

affects the end-to-end delay along with distance $(r)$ and channel access probability $(p)$. Increase in node density increases the number of nodes in the region as well as number of contending nodes. The effect of node density is analysed in steps of 50, 150 and 200 to analyse the end-to-end behaviour along with $r$ and $p$.

Figure 6 shows the 3D plot of end-to-end delay with variation in $p$ and $r$ fixing $\Lambda=50$ and $\lambda=0.025$. The optimal region for minimal end-to-end delay is a valley as shown in the plot. End-to-end delay with and without silent 
relay nodes is plot in Figures 6(b) and 6(a) respectively. From the figures the minimal delay region is very less with silent relay nodes compared to without silent relay nodes. A bound on end-to-end delay is fixed to 30 to find out optimal transmission distance. The distance $(r)$ is [ $\left[\begin{array}{ll}0 & 0.9\end{array}\right]$ without silent relay node and [ $\left[\begin{array}{ll}0 & 0.8\end{array}\right]$ with silent relay nodes. The reduction in transmission distance with silent relay nodes is less compared to without silent relay nodes. This reduction in distance $(r)$ is due to the fact that silent relay nodes are being silent most of the time than forwarding data to the next hop.

Node density $(\Lambda)$ is increased to 150 as shown in Figure 7. Simulations are carried out for both cases with and without silent relay nodes as shown in Figures 7(a) and 7(b).
Same end-to-end delay bound of 30 units is taken for $\Lambda=150$ also. The transmission distance $(r)$ is [0 0.8$]$ without silent relay nodes as shown in Figure 7(a), where as the minimal end-to-end delay starts at 50 units with silent relay nodes as shown in Figure $7(\mathrm{~b})$. Thus there is no optimal transmission distance with silent relay nodes when the end-to-end delay bound is 30 units. The node density is further increased to 200 to observe the end-to-end delay results as shown in Figure 8. The minimum end-to-end delay for $\Lambda=200$ is more than 30 units in both the cases of with and without silent relay nodes as shown in Figures 8(a) and 8(b).

Figure 7 3D plot of end to end delay with variation in distance $(r)$ and channel access probability $(p)$ along with, (a) $\Lambda=150, \lambda=0.025$, $\Delta=0$ without silent relay nodes b) $\Lambda=150, \lambda=0.025, \Delta=1, \alpha=0.2$ with silent relay nodes (see online version for colours)

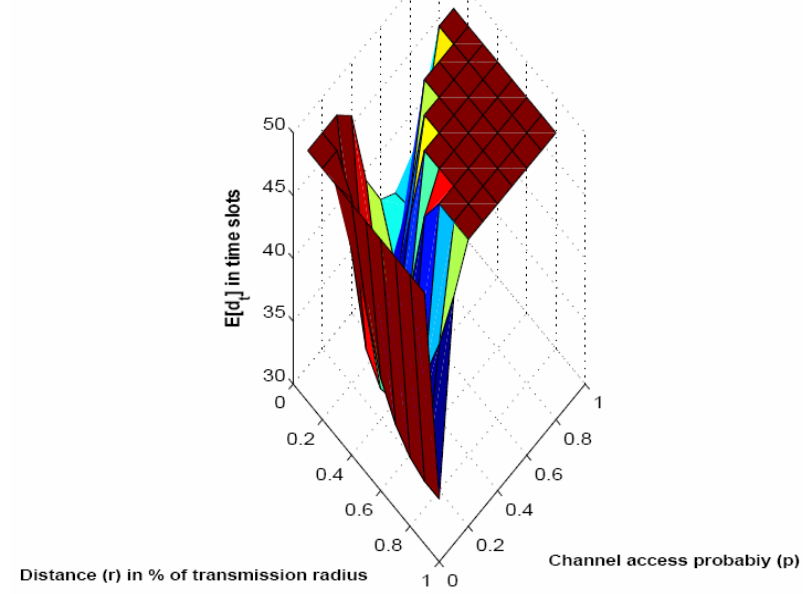

(a)
7 (b) $E\left[d_{1}\right]$ (with silent relay nodes) vs $p \& r, \Lambda=150, \lambda=0.025, \Delta=1, \alpha=0.2$

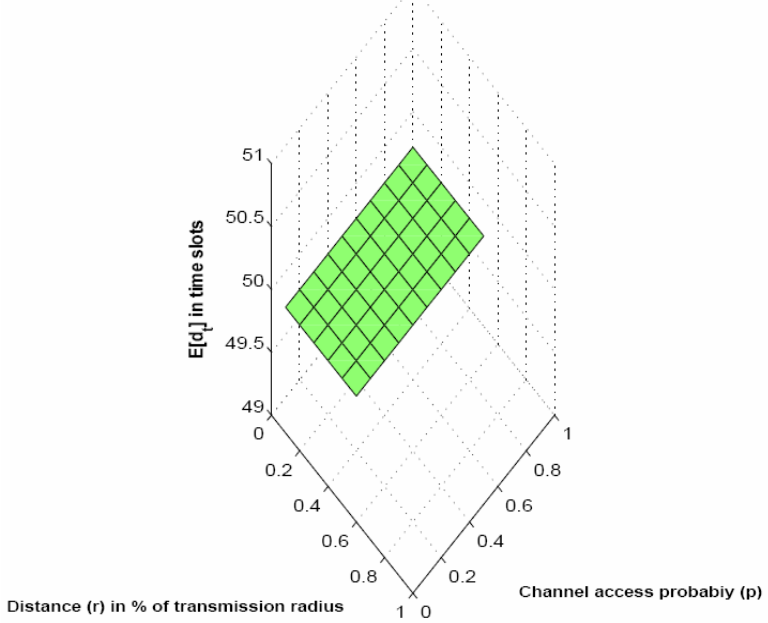

(b)

Figure 83 p plot of end to end delay with variation in distance $(r)$ and channel access probability ( $p$ ) along with, (a) $\Lambda=200, \lambda=0.025$, $\Delta=0$ without silent relay nodes b) $\Lambda=200, \lambda=0.025, \Delta=1, \alpha=0.2$ with silent relay nodes (see online version for colours)

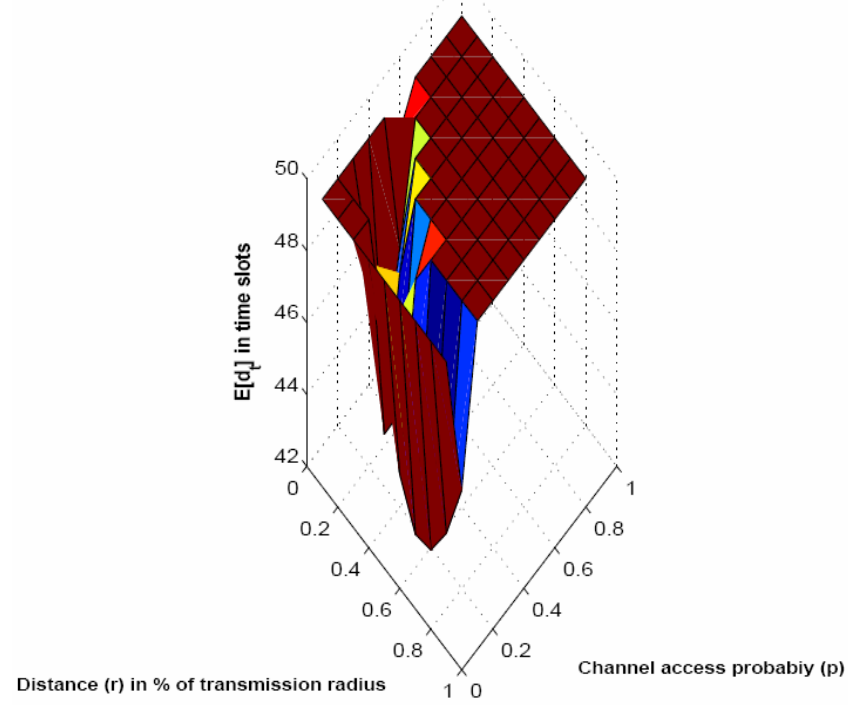

(a)

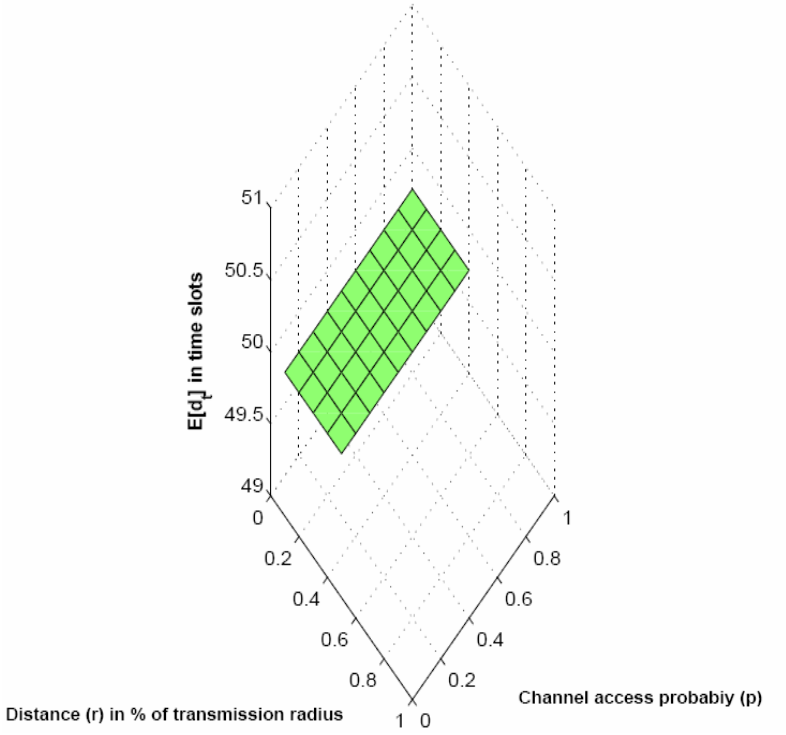

(b) 
Figure $93 \mathrm{D}$ plot of end to end delay with variation in distance $(r)$ and channel access probability $(p)$ along with, (a) $\Lambda=100, \lambda=0.01$, $\Delta=0$ without silent relay nodes b) $\Lambda=100, \lambda=0.01, \Delta=1, \alpha=0.2$ with silent relay nodes (see online version for colours)

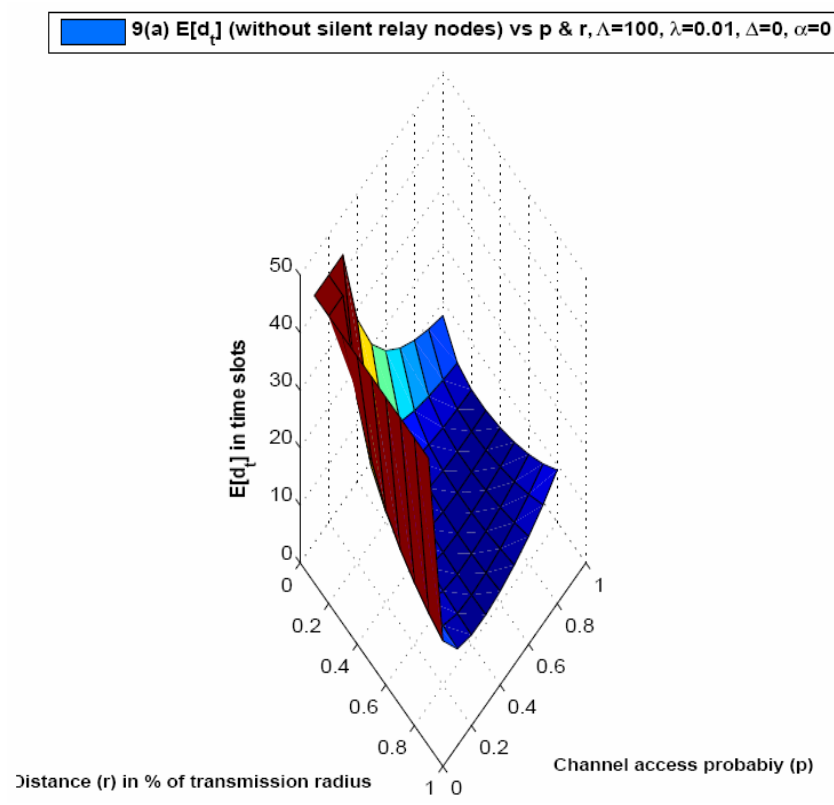

(a)

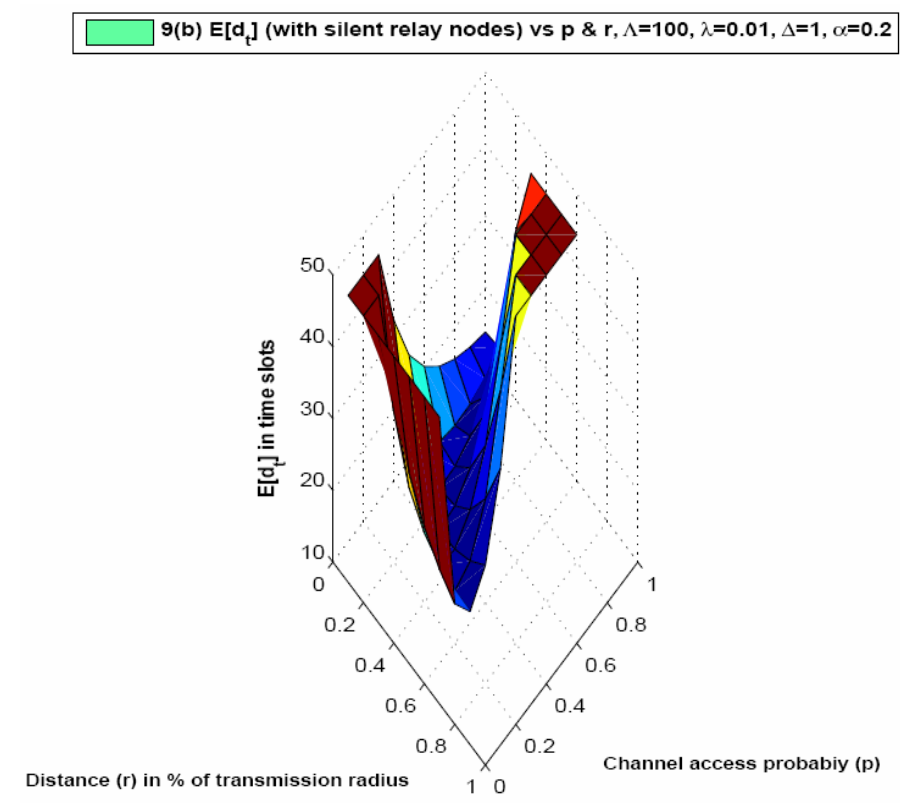

(b)

Minimum end-to-end delay is increasing with silent relay nodes. Minimum end-to-end delay region is reducing with a steeper slope with silent relay node case as shown in Figures 6(b), 7(b) and 8(b). The minimal delay region becomes almost zero, for $\Lambda=200$ without relay nodes but with silent relay nodes it is zero at $\Lambda=150$ itself. The observations shows that node density is a dominant factor which affects end-to-end delay. Thus effect of node density is analysed with silent relay nodes in this section. End-to-end delay with consideration of throughput effect along with silent relay nodes is discussed in the next subsection.

\subsection{Effect of throughput on end-to-end delay with silent relay nodes}

End-to-end delay is also a function of throughput $(\lambda)$. Analysis of throughput effect on end-to-end delay is discussed in this section. Throughput $(\lambda)$ is transmission of bits to the destination from source. These bits should be forwarded through relay nodes in a multi-hop network. If the throughput $(\lambda)$ is more the relay nodes need more time to transfer data to the destination which will create more end-to-end delay. The effect of silent relay nodes will further increase the end-to-end delay as the relay nodes supposed to forward are silent due to interference.

The total end-to-end delay with variation in distance $(r)$ and channel access probability $(p)$ is simulated by keeping node density $(\Lambda)$ constant at 100 and throughput is varied between .01 and .03 as shown in Figures 9 and 10

respectively. End-to-end delay simulations show that the minimal delay region of end-to-end delay is reducing with increase in the throughput without consideration of silent relay node delay effect as shown in Figures 9(a) and 10(a). A bound of 30 units end-to-end delay will result in transmission distance of [ $\left[\begin{array}{ll}0 & 0.8\end{array}\right]$ and $\left[\begin{array}{ll}0 & 0.6\end{array}\right]$ respectively for with and without silent relay nodes as shown in Figures 9(a) and 9 (b). Transmission distance for the same delay bound of 30 reduced to $25 \%$ of without relay nodes. In a similar fashion, when the throughput is increased to 0.03, keeping other parameters same, it is observed that the minimal end-to-end delay is 50 units, and for bound of 30 units there is no optimal distance as shown in Figure 10(b). Increase in throughput $(\lambda)$ is reducing the minimal end-to-end delay region drastically. The optimal end-to-end delay region for $\lambda=0.03$ is considerable as shown in Figure 10(a), where as it is zero with silent relay nodes in Figure 10(b). The reason being overlap of relay transmission regions increases the number of nodes to be silent to double compared to non-overlap region. When the throughput requirement is more, the time it takes to send more amount of data increases as the number of nodes in silent are more. If the relay nodes in the hoping region belong to overlapping region, these nodes remain silent instead of forwarding data to next hop.

Thus in this section the effect of silent relay nodes on the end-to-end delay is extensively analysed. 
Figure $103 \mathrm{D}$ plot of end to end delay with variation in distance $(r)$ and channel access probability ( $p$ ) along with, (a) $\Lambda=100, \lambda=0.03$, $\Delta=0$ without silent relay nodes b) $\Lambda=100, \lambda=0.03, \Delta=1, \alpha=0.2$ with silent relay nodes (see online version for colours)

10(a) $E\left[d_{t}\right]$ (without silent relay nodes) vs $p \& r, \Lambda=100, \lambda=0.03, \Delta=0, \alpha=0$

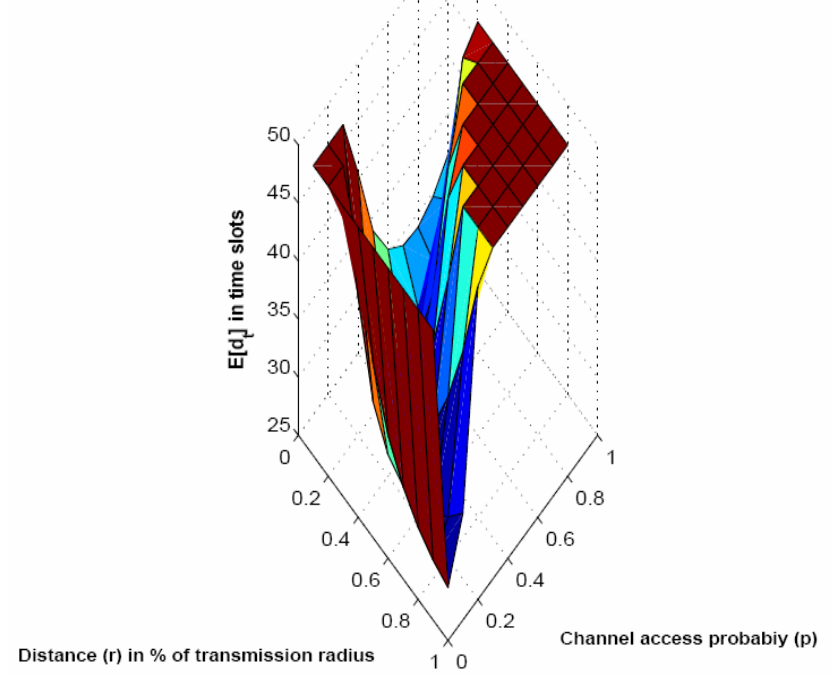

(a)

\section{Effect of increase in transmit power on end-to-end delay}

Transmit power increment reduces number of hops and end-to-end delay but increases interference among neighbour nodes. Number of interfering nodes can be reduced by implementing interference model which says that all other neighbour nodes should remain silent for a transmission in its transmission region. In the case of simultaneous multi-hop transmissions, transmission of one route cause other relay nodes to remain silent which we define as silent relay nodes. The number of silent relay nodes increases with increase in transmission power of one or more nodes of a multi-hop route. Hence end-to-end delay does not decrease with increment in transmission power due to consideration of above interference model. Increase in transmission power is simulated in this paper by analogously increasing the neighbourhood transmission region to 1,2 and 3 respectively and results are as shown in Figures 11 to 13 . Along with the transmission radius other factors of consideration are node density $(\Lambda)$, channel access probability $(p)$ and throughput $(\lambda)$. The detailed analysis of the results are followed in the next paragraphs.

$$
(\lambda * D) / r \leq P(\text { Successful transmission })
$$

Results are plotted in Figures 11 to 13. Figure 11 plots end-to-end delay versus increase in transmit power for different network parameters. Effect of increase in transmission power or neighbourhood region is analysed varying node density in steps of $20 \%$ and fixing throughput at constant value. Channel access probability is varied in steps of 0.05 units to get a sub figure result as shown in Figure 11. Each sub-figure in Figure 11 has variation of channel access probability with throughput being constant. 10(b) $E\left[d_{t}\right]$ (with silent relay nodes) vs $p \& r, \Lambda=100, \lambda=0.03, \Delta=1, \alpha=0.2$

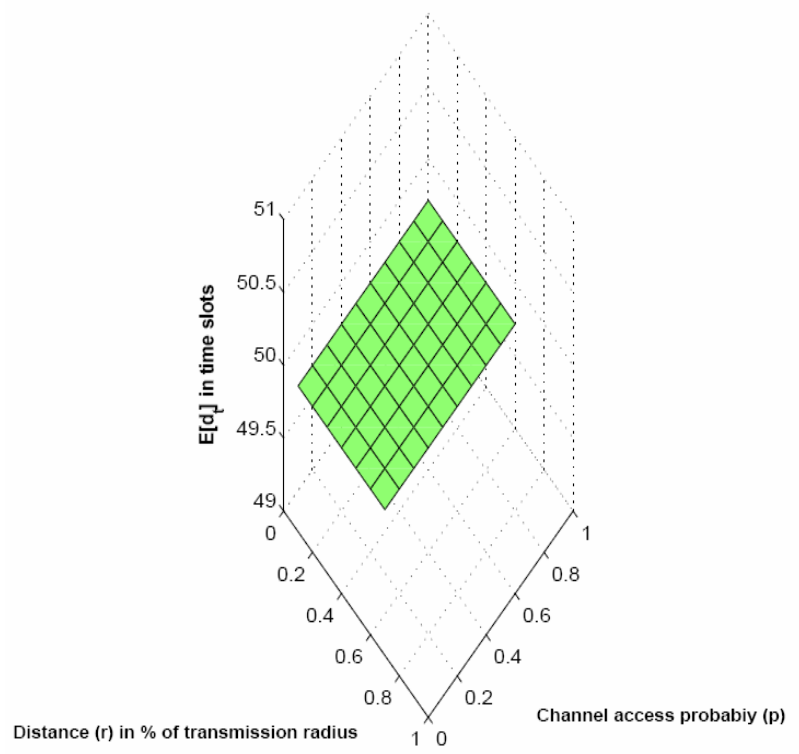

(b)

Effect of varying throughput is plotted in Figures 11 to 13. Some network parameters are fixed to simplify the analysis, rather than simultaneously varying all parameters. End-to-end delay is decreasing for increase in transmit power up to two neighbourhood regions only. From Figure 11 it is seen that low node density cases (20 to 40\%) has reduced end-to-end delay even after two hops neighbourhood region. The decrease of end-to-end delay is very less for high density because when node density is high even though transmit power is increased the number of nodes to remain silent are more according to interference model. As a result end-to-end delay observed to be more. The end-to-end delay is 180 time units in sub Figure 1 of Figure 11 for channel access probability of 0.05 . Maximum value of end-to-end delay is 250 time units in sub Figure 4 of Figure 11. Its been observed from Figures 11 to 13 some of the curves are ending abruptly as the stability condition shown in equation (14) is not met for the network parameters such as node density, throughput and channel access probability values.

If we closely compare the sub-figures of Figure 11 where channel access probability is increased in steps of 0.05 units. It is observed that for channel access probability of 0.1 the end-to-end delay is less than 0.05 case. This behaviour is due to low channel access probability of the network. Low access probability will result in more number of attempts which in turn increase the end-to-end delay. Increase in channel access probability will increase number of collisions which again increases delay. This result is only observed in Figure 11, but if we compare Figures 11 to 13 the end-to-end delay is increasing with increase in channel access probability. End-to-end delay is increasing with increase in throughput because increase in throughput is dominating than change in channel access probability. 
Figures 11 to 13 are results obtained by changing throughput values from $0.01,0.025$ to 0.03 units respectively. End-to-end delay is increasing with increase in channel access probability in each figure. The ranges of end-to-end delay are different for different channel access probabilities and throughput. Maximum end-to-end delay in last sub figures of Figures 11 to 13 are 250, 5,000 and 14,000 time units. End-to-end delay curves slopes are increasing exponentially with throughput from Figures 11 to 13 . Throughput is observed to be dominant factor as average throughput increases the number of nodes that want channel access increases. Even though transmit power increases the end-to-end delay is increased due to the interference model. More throughput requirement demands more nodes to contend for the channel in order to deliver more data, however interference model does not allow more nodes to transmit to avoid collisions. Increase in transmit power is feasible in terms of allowable end-to-end delay and from simulation results it is observed that increment in transmit power is feasible up to twice the power or one hop neighbourhood.

\section{Conclusions}

End-to-end delay is one of the important quality of service metric considered in wireless ad hoc network. Effect of transmission radius $(r)$, channel access probability $(p)$, node density $(\Lambda)$, and throughput $(\lambda)$ are studied in the literature. Effect of silent relay nodes delay in dense overlapping transmission regions is analysed in this paper. The simulation results show that the overlap of relay node transmission regions in dense network creates more end-toend delays. The number of nodes to remain silent is increased to decrease the interference among nodes.

End-to-end delay is analysed with respect to transmission radius $(r)$, channel access probability $(p)$ by considering node density $(\Lambda)$, throughput $(\lambda)$ and percentage of silent relay nodes $(\alpha)$. Results show that end-to-end delay is more with silent relay nodes compared to without silent relay nodes. The node density and throughput parameters are varied one at a time to investigate the effect on end-to-end delay. The simulation results show that silent relay nodes which have common transmission region is affecting considerably the total end-to-end delay by reducing the optimal minimal region of end-to-end delay. The effect of node density $(\Lambda)$ is dominant with respect to throughput changes considering silent relay nodes as shown in the simulation results. Effect on end-to-end delay with increase in transmission power to next neighbourhood regions is analysed with network parameters like node density, throughput and channel access probability. Results show that increase in transmission power to reduce number of hops is feasible up to one or two neighbourhood regions after which the end-to-end delay is increasing exponentially. The parameters considered for the analysis are interdependent. Exact expression for end-to-end delay by considering interdependencies among the parameters are future scope of research.

Figure 11 Total end-to-end delay with variation in transmit power and channel access probability $(p), \lambda=0.01$ (see online version for colours)
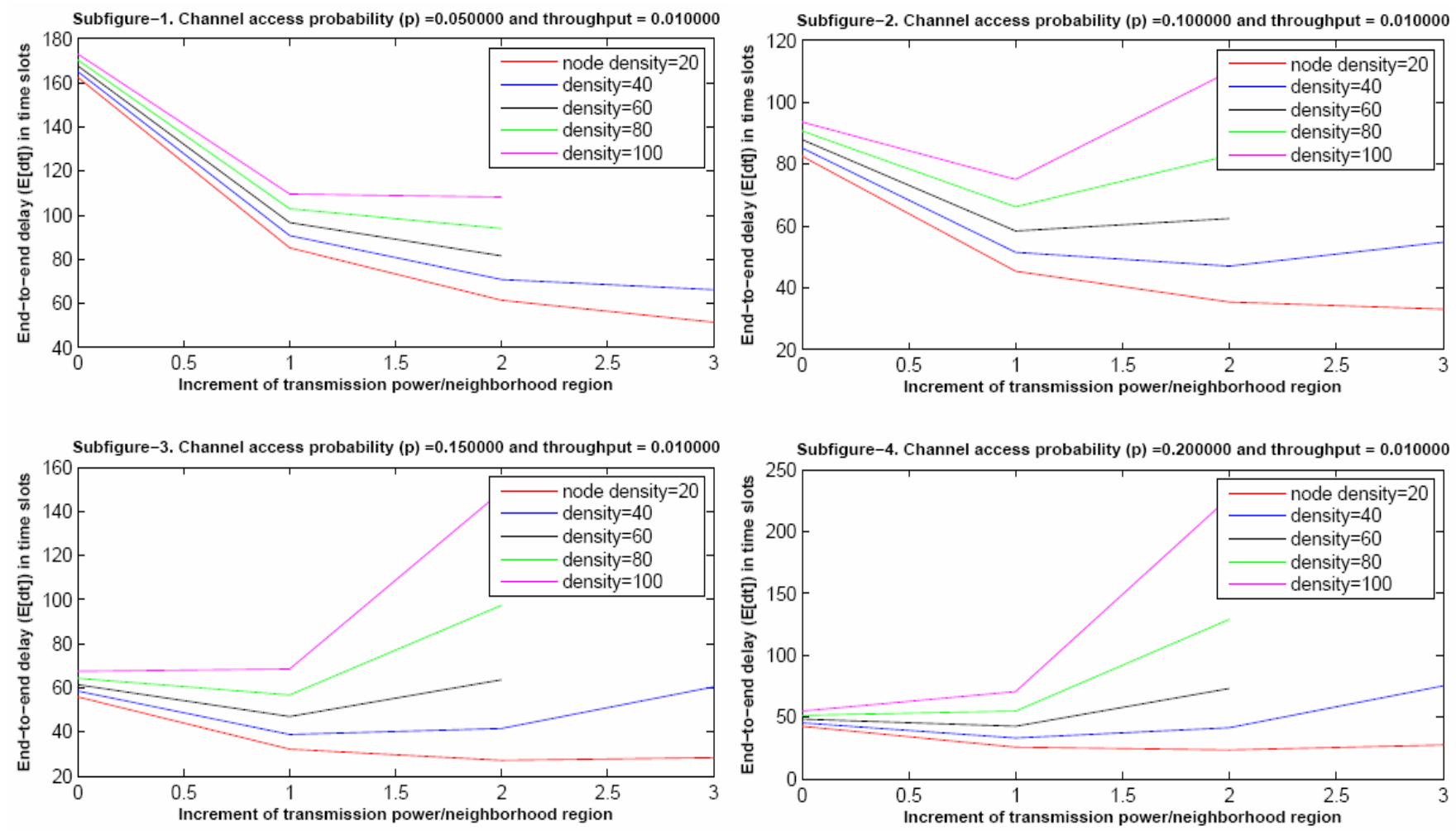
Figure 12 Total end-to-end delay with variation in transmit power and channel access probability $(p), \lambda=0.025$ (see online version for colours)
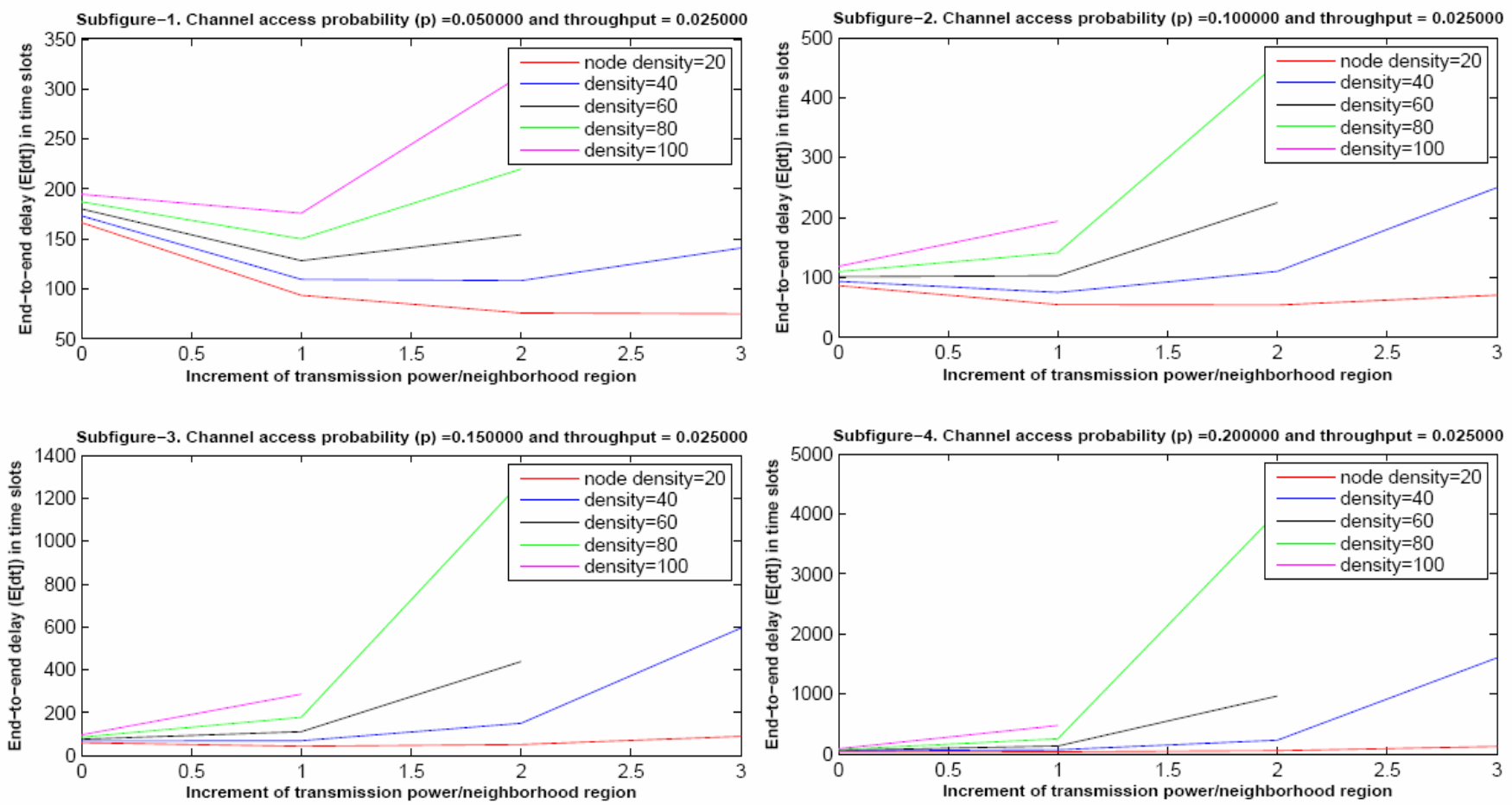

Figure 13 Total end-to-end delay with variation in transmit power and channel access probability $(p), \lambda=0.03$ (see online version for colours)
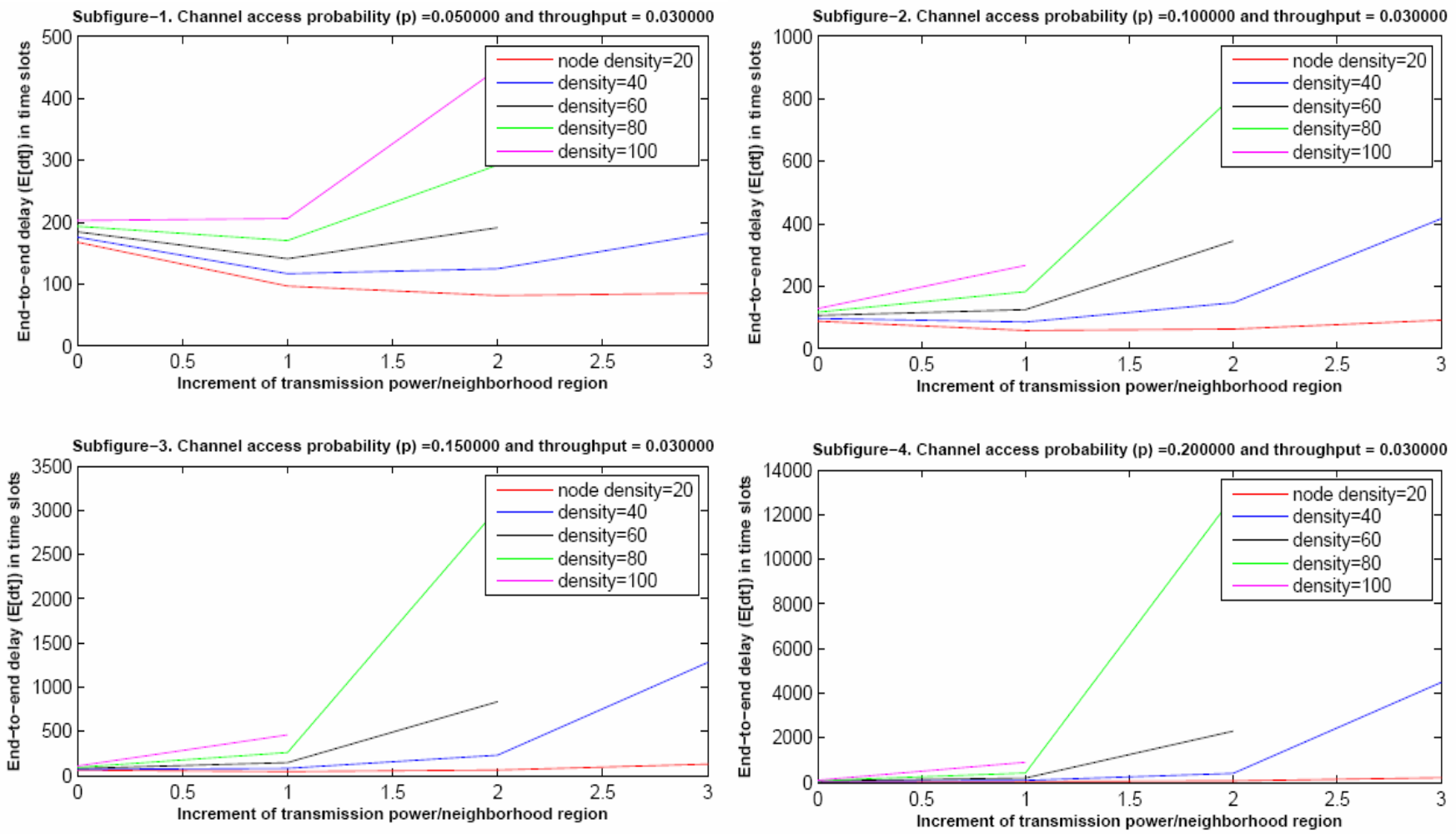


\section{References}

El Gamal, A., Mammen, J., Prabhakar, B. and Shah, D. (2004) 'Throughput-delay trade-off in wireless networks', Proc. INFOCOM.

$\mathrm{Fu}, \mathrm{W}$. and $\mathrm{Ma}, \mathrm{J}$. (2009) 'Implementation and verification of power management scheme over indoor multipath channel at UHF bands', Integrated Circuits, ISIC '09, Proceedings of the 12th International Symposium.

Gomez, J., Campbell, A.T., Naghshineh, M. and Bisdikian, C. (2003) 'Suporting dynamic power controlled routing in wireless ad hoc networks', ACM/Kluwer Journal on Wireless Networks (WINET), to appear.

Grossglauser, M. and Tse, D. (2001) 'Mobility increases the capacity of ad hoc networks', Proc. INFOCOM.

Gupta, P. and Kumar, P.R. (2000) 'The capacity of wireless networks', IEEE Transactions on Information Theory, March, Vol. 46, No. 2.

Heinzelman, W., Chandrakasan, A. and Balakrishnan, H. (2000) 'Energy-efficient communication protocol for wireless microsensor networks', in Proc. HICSS, January.

Kar, K., Kodialam, M., Lakshmanan, T.V. and Tassiulas, L. (2003) 'Routing for network capacity maximization in energy-constrained ad hoc networks', Proc. INFOCOM, April.

Kawadia, V. and Kumar, P.R. (2003) 'Power control and clustering in ad-hoc networks', Proc. INFOCOM, April.

Kawadia, V., Narayanaswamy, S., Sreenivas, R.S., Rozovsky, R. and Kumar, P.R. (2001) 'Protocols for media access control and power control in wireless networks', in 40th IEEE Conference on Decision and Control, December, pp.1935-1940.

Lin, S., Zhang, J., Gu, L., He, T. and Stankovic, J. (2006) 'ATPC: adaptive transmission power control for wireless sensor networks', in Proceedings of SenSys '06, November.

Narasimhan, S. and Kunniyur, S. (2005) 'Modelling the effect of network parameters on delay in wireless ad-hoc networks', IEEE SECON, 26-29 September, pp.340-349.
Oh, S-H. (2012) 'TPC-BS: transmission power control based on binary search in the wireless sensor networks', Sensors Applications Symposium (SAS).

Palmieria, F. and Castiglione, A. (2012) 'Condensation-based routing in mobile ad-hoc networks', Journal of Mobile Information Systems, Vol. 8, No. 3, pp.199-211, DOI: 10.3233/MIS-2012-0140, IOS Press.

Pianegiani, F. (2011) 'miniTP: a protocol for the minimization of the transmit power in wireless networks', Instrumentation and Measurement Technology Conference (I2MTC), IEEE, Binjiang.

Rozovsky, R. and Kumar, P.R. (2001) 'SEEDEX: a MAC protocol for ad hoc networks', in Proceedings of the ACM Symposium on Mobile Ad Hoc Networking and Computing, MobiHoc, October.

Rukpakavong, W., Phillips, I. and Guan, L. (2011) 'Neighbour Discovery for transmit power adjustment in IEEE 802.15.4 using RSSI', New Technologies, Mobility and Security (NTMS), 4th IFIP International Conference, Paris.

Singh, S. and Raghavendra, C.S. (1998) 'Power efficient MAC protocol for multihop radio networks', Proc IEEE International Symposium on Personal, Indoor and Mobile Radio Communications.

Son, D., Krishnamachari, B. and Heidemann, J. (2004) 'Experimental study of the effects of transmission power control and blacklisting in wireless sensor networks', IEEE SECON, October.

Ye, W., Heidemann, J. and Estrin, D. (2002) 'An energy-efficient MAC protocol for wireless sensor networks', Proc. of the 21st International Annual Joint Conference of the IEEE Computer and Communications Societies, June.

Yerra, R.V.P. and Rajalakshmi, P. (2013) 'Effect of relay nodes on end-to-end delay in multi-hop wireless ad hoc networks', Advanced Information Networking and Applications Workshops (WAINA) 27th International Conference on Advanced Information Networking and Applications (AINA-2013), pp.343-348, Barcelona, Spain, DOI: 10.1109/WAINA.2013.181. 